Document downloaded from:

http://hdl.handle.net/10251/176448

This paper must be cited as:

Parra Gómez, J.; Olivares-Sánchez-Mellado, I.; Ramos, F.; Sanchis Kilders, P. (2020). Ultracompact non-volatile Mach-Zehnder switch enabled by a high-mobility transparent conducting oxide. Optics Letters. 45(6):1503-1506. https://doi.org/10.1364/OL.388363

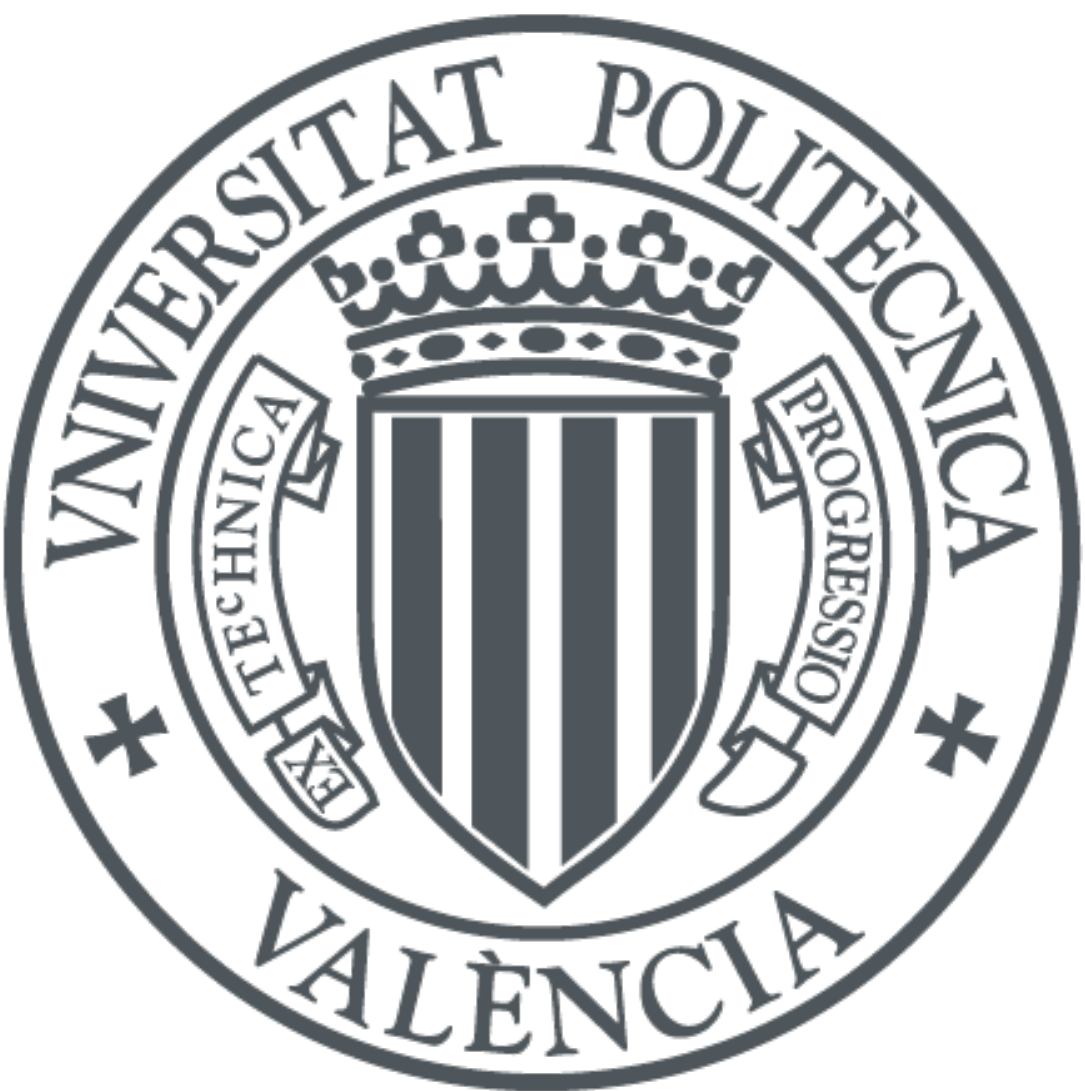

The final publication is available at

https://doi.org/10.1364/OL.388363

Copyright The Optical Society

Additional Information 


\title{
Ultra-compact non-volatile Mach-Zehnder switch enabled by a high-mobility transparent conducting oxide
}

\author{
Jorge Parra ${ }^{1}$, Irene Olivares ${ }^{1}$, Francisco Ramos ${ }^{1}$, and Pablo Sanchis ${ }^{1}$,* \\ ${ }^{1}$ Nanophotonics Technology Center, Universitat Politènica de València, Camino de Vera s/n, 46022 Valencia, Spain \\ *Corresponding author: pabsanki@ntc.upv.es
}

Compiled February 24, 2020

Compact and broadband non-volatile silicon devices are mainly absorption-based. Hence, access to low-loss non-volatile phase shifters is still a challenge. Here, this problem is addressed by using a high-mobility transparent conducting oxide such as cadmium oxide as a floating gate in a flash-like structure. This structure is integrated in a Mach-Zehnder interferometer switch. Results show an active length of only $30 \mu \mathrm{m}$ to achieve a $\pi$ phase shift. Furthermore, an extinction ratio of $20 \mathrm{~dB}$ and insertion loss as low as $1 \mathrm{~dB}$ may be attained. The device shows an optical broadband reponse and can be controlled with low-power pulses in the nanosecond range. These results open a new way for enabling compact silicon based phase shifters with non-volatile performance. (๑) 2020 Optical Society of America

\section{http://dx.doi.org/10.1364/ao.XX.XXXXXX}

Non-volatile silicon photonics is currently a hot-topic in the field of photonic integrated circuits (PICs). The ability of providing a semi-permanent change in the effective refractive index after applying an stimulus to this kind of waveguide can be useful for enabling new and emerging applications. Memory effect can be taken in advantage in disruptive applications such as neural networks [1], reconfigurable photonics [2] or photonic memories [3]. Furthermore, non-volatility can be provided either by integrating phase change materials such as $\mathrm{Ge}_{2} \mathrm{Sb}_{2} \mathrm{Te}_{5}$ (GST) [4] or by using the same concept as in electronic memories based in a floating gate [5-8].

The field of non-volatile absorption-based devices is mainly covered by the utilization of GST. The large change in the imaginary part of its refractive index between the amorphous and crystalline state allows very compact devices with broadband response [9]. However, most of emerging applications rely on phase devices. Therefore, phase change materials are not suitable to be used for low-loss non-volatile phase applications. On the other hand, floating gate based devices exploit free carrier dispersion effects of the materials comprising the non-volatile structure. Thus, silicon plasma dispersion effect of the waveguide can be exploited to induce a phase shift. However, the change of the silicon refractive index with carriers is very weak
[10]. Hence, long waveguides or resonant structures such as microring resonators with inherit narrow bandwidth are required [7].

Recently, we proposed an ultra-compact and broadband electro-absorptive memory by using a transparent conducting oxide (TCO) such as indium tin oxide (ITO) as a floating gate [11]. Indium tin oxide has been proposed as an active material due to its epsilon-near-zero (ENZ) regime [12-15]. However, ITO could not be suitable for phase applications due to high optical losses and thus, other TCOs such as cadmium oxide (CdO) has been proposed for this purpose [16-18].

In this Letter, we propose to replace the ITO floating gate by $\mathrm{CdO}$ in order to reduce optical loss and thus, enable a low-loss non-volatile phase shifter. The capabilities of this new device are also investigated for optical switching. In this context, we propose a non-volatile Mach-Zehnder interferometer (NVMZI) switch.

The proposed non-volatile switch is illustrated in Fig. 1. It is composed of a $2 \times 2$ symmetric NVMZI. Both branches have an electro-optic structure that induces a phase shift with a

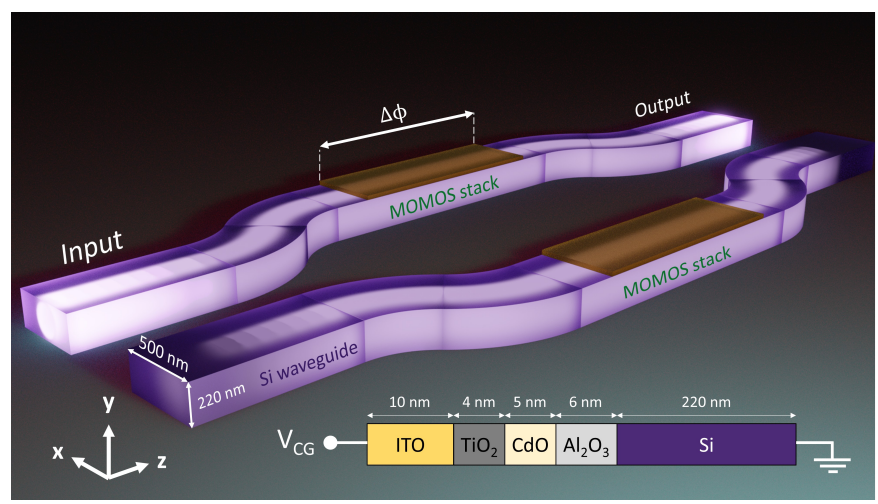

Fig. 1. Illustration of the proposed non-volatile Mach-Zehnder interferometer (NVMZI) switch. The NVMZI is comprised of a $500 \times 220 \mathrm{~nm}^{2}$ silicon waveguide fully surrounded of $\mathrm{SiO}_{2}$. The phase shift is induced by one of the MOMOS arms. The materials and thickness used for the MOMOS layers are shown in the inset. 

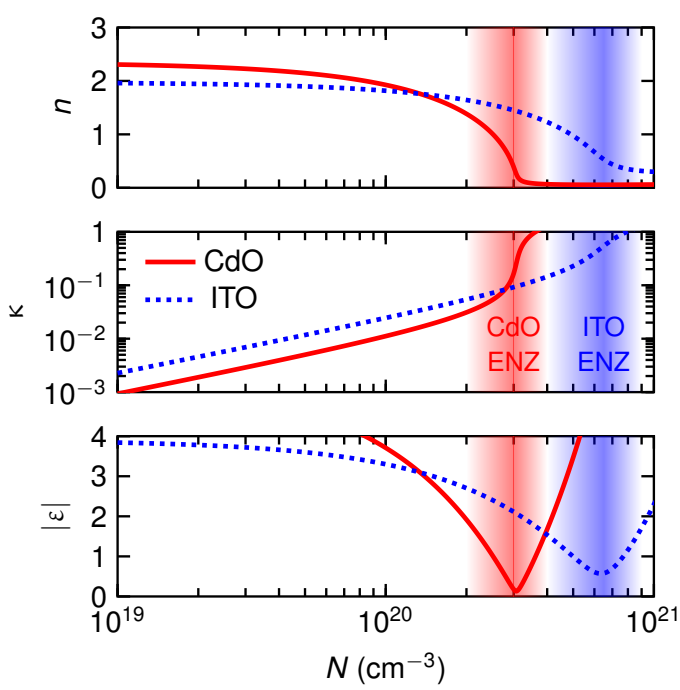

Fig. 2. Refractive index, $n+j \kappa$, and absolute value of the permittivity, $|\varepsilon|$, of $\mathrm{CdO}$ and ITO as a function of the free carrier concentration, $N$, at $\lambda=1550 \mathrm{~nm}$. Optical properties were calculated using the Drude model and parameters from [18, 19].

non-volatile effect. The electro-optic structure is comprised of a metal-oxide-metal-oxide-semiconductor (MOMOS) stack. A low-doped ITO layer on top is electrically contacted whereas the silicon waveguide is grounded. The floating gate, i.e., the sandwiched active layer, is either charged or discharged through the $\mathrm{Al}_{2} \mathrm{O}_{3}$ tunneling oxide by applying positive or negative voltage pulses, respectively. As a result, due to the free carrier dispersion effect of the floating gate, this undergoes a change in its refractive index and thus, induces a phase change in the optical mode. The materials and the thicknesses used for the MOMOS layers are depicted in the inset of the Fig. 1. Further details of the MOMOS structure and methods can be found in our previous work [11].

Transparent conducting oxides are ideal candidates to be used as a floating gate since they exhibit a strong free carrier dispersion effect in the near-infrared because the ENZ regime [12]. The permittivity, $\varepsilon=\varepsilon^{\prime}+j \varepsilon^{\prime \prime}$, of TCOs in the near-infrared is described by the Drude model:

$$
\varepsilon=\varepsilon_{\infty}\left(1-\frac{\omega_{p}^{2}}{\omega^{2}+j \omega \Gamma}\right),
$$

where $\varepsilon_{\infty}$ is the high-frequency permittivity, $\omega$ is the angular frequency, $\Gamma$ is the damping factor and $\omega_{p}$ is the plasma frequency, which can be expressed as:

$$
\omega_{p}=\sqrt{\frac{N q^{2}}{\varepsilon_{\infty} \varepsilon_{0} m^{*}}} .
$$

The parameter $N$ stands for the free carrier concentration, $q$ is the electron charge, $\varepsilon_{0}$ is the vacuum permittivity and $m^{*}$ is the effective electron mass. Interestingly, the TCO optical response can be drastically changed by accumulating carriers. In terms of refractive index, $(\sqrt{\varepsilon})$, the real part can be lowered up to one order of magnitude. Furthermore, this large change is boosted thanks to the light-matter interaction (LMI) enhancement that occurs because the ultra-low value of $|\varepsilon|$ [15]. However, this change is usually accompanied with a large increase in the optical losses.

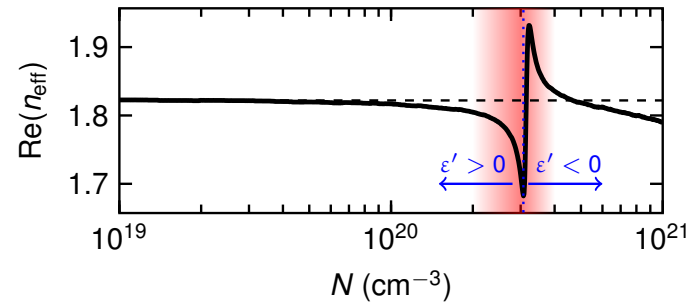

(a)

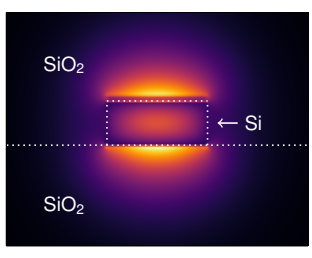

(b)

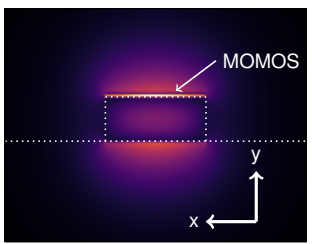

(c)
Fig. 3. (a) Real part of the effective refractive index, $n_{\mathrm{eff}}$, for TM polarization as a function of the $\mathrm{CdO}$ floating gate free carrier concentration, $N$. Dashed line stands for the reference $n_{\text {eff }}$ and dotted line for $\varepsilon^{\prime}=0$ of $\mathrm{CdO}$. $(\mathrm{b}, \mathrm{c})$ Electric field profile, $\left|E_{y}\right|$, of the MOMOS cross-section for different carrier concentration in the CdO floating gate: (b) $2 \times 10^{19} \mathrm{~cm}^{-3}$ and (c) $2.3 \times 10^{20} \mathrm{~cm}^{-3}$.

Optical losses of a material are associated with $\varepsilon^{\prime \prime}$. From Eq. $1, \varepsilon^{\prime \prime}$ of TCOs can be expressed as:

$$
\varepsilon^{\prime \prime}=\frac{\Gamma \varepsilon_{\infty} \omega_{p}^{2}}{\omega\left(\omega^{2}+\Gamma^{2}\right)},
$$

which shows that losses are strongly correlated to $\Gamma$. The damping factor is inversely proportional to the mobility of the material $(\Gamma \propto 1 / \mu)$. Therefore, the mobility of the TCO should be as high as possible to obtain the minimum optical loss. Among the TCOs, CdO stands out with reported mobility values up to 1000 $\mathrm{cm}^{2} \mathrm{~V}^{-1} \mathrm{~s}^{-1}$ [20]. Conversely, ITO exhibits mobilities of around $10 \mathrm{~cm}^{2} \mathrm{~V}^{-1} \mathrm{~s}^{-1}$ [19]. Thus, $\mathrm{CdO}$ is as a better active material in the floating gate. In this work, $\mathrm{CdO}$ values from [18] were used for optical and electronic simulations: $\varepsilon_{\infty}=5.5, \mu=500 \mathrm{~cm}^{2}$ $\mathrm{V}^{-1} \mathrm{~s}^{-1}, m^{*}=0.12 m_{e}$ and electron affinity of $4.51 \mathrm{eV}$.

The complex refractive index, $n+j \kappa$, and the absolute value of permittivity, $|\varepsilon|$, as a function of $N$ for both materials are shown in Fig. 2. The ENZ region of $\mathrm{CdO}$ is found around $3 \times 10^{20} \mathrm{~cm}^{-3}$, whereas for ITO is at $6.5 \times 10^{20} \mathrm{~cm}^{-3}$ due to the higher electron effective mass. The lower value of $\Gamma$ of $\mathrm{CdO}$ has an impact in both real and imaginary parts of the refractive index as well as in the $|\varepsilon|$ value. On one hand, $n$ of $\mathrm{CdO}$ presents stepper and larger change in comparison with ITO in the ENZ regime. Furthermore, $\kappa$ is one order of magnitude lower than ITO when the ENZ state begins to undergo. On the other hand, $\mathrm{CdO}$ presents higher LMI since the minimum value of $\varepsilon$ is fivefold reduced with respect to ITO. As a result, this combination of the large change in $n$, low- $\kappa$ and higher LMI could allow using $\mathrm{CdO}$ as a floating gate for low-loss and compact non-volatile phase shifters.

Finite element method (FEM) based simulations were carried out for the cross-section of the MOMOS structure. The effective refractive index was obtained by sweeping the free carrier concentration of the $\mathrm{CdO}$ floating gate between $10^{19} \mathrm{~cm}^{-3}$ and $10^{21}$ $\mathrm{cm}^{-3}$. Then, the length to obtain a $\pi$ phase shift, $L_{\pi}$, and the 

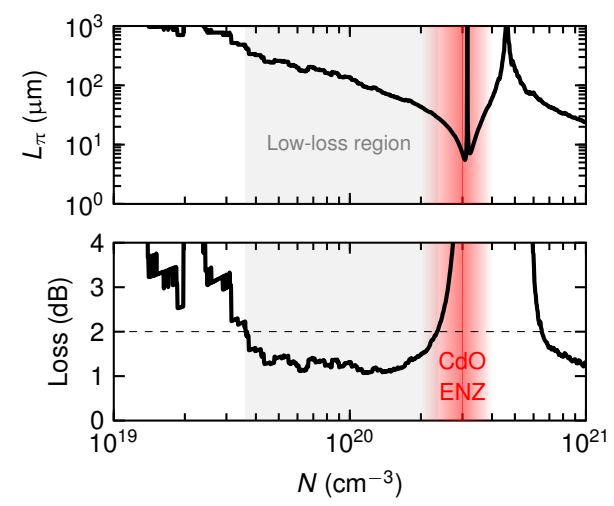

Fig. 4. MOMOS length to achieve a $\pi$ phase shift, $L_{\pi}$, and associated optical loss as a function of the $\mathrm{CdO}$ floating gate free carrier concentration $(N)$ at $\lambda=1550 \mathrm{~nm}$.

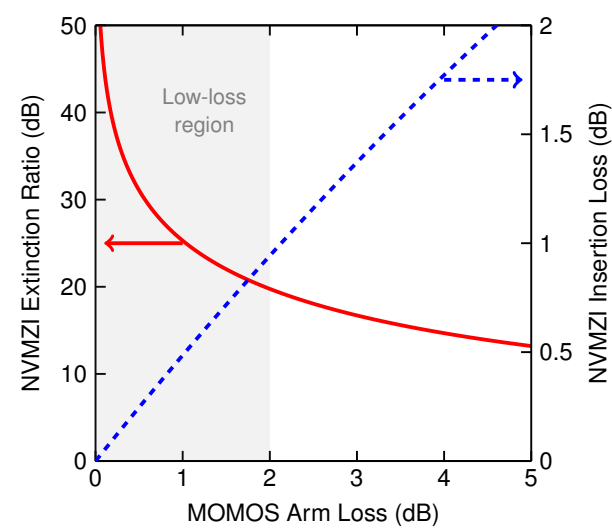

Fig. 5. Extinction ratio and insertion loss of the NVMZI by changing the losses of the MOMOS at $\lambda=1550 \mathrm{~nm}$.

associated loss were calculated. The value of $L_{\pi}$ is given as:

$$
L_{\pi}=\frac{\lambda}{2\left|\Delta n_{\mathrm{eff}}\right|},
$$

where $\lambda$ is the working wavelength and $\Delta n_{\text {eff }}$ is the increment in the real part of the effective refractive index taking as a reference value the obtained for $N=2 \times 10^{19} \mathrm{~cm}^{-3}$.

The variation of the real part of the effective refractive index with $N$ is depicted in Fig. 3a. The Fano-like shape is induced by the change in the sign of real part of the permittivity [21]. Both minimum and maximum values are found in the ENZ region. The minimum corresponds to a photonic mode since $\varepsilon^{\prime}$ of $\mathrm{CdO}$ is positive, whereas the maximum is an hybrid-plasmonic mode due to $\varepsilon^{\prime}<0$. For low values of $N$, the typical TM mode profile of a silicon waveguide is obtained (Fig. 3b). However, as the $\mathrm{CdO}$ approximates to the ENZ regime, the light shifts to the floating gate and confines mostly within it (Fig. 3c). Ultimately, for higher values of $N, \mathrm{CdO}$ behaves as a metal and a plasmonic mode is obtained.

Fig. 4 shows $L_{\pi}$ and the optical loss as a function of $N$. As the free carrier concentration is increased from low values to the ENZ region, the greater the difference in the effective refractive index and thus, the lower the value of $L_{\pi}$. Two ideal infinity values for $L_{\pi}$ are achieved because of the Fano-like shape of $\operatorname{Re}\left(n_{\mathrm{eff}}\right)$. The first one, during the transition of $\mathrm{CdO}$ from dielectric $\left(\varepsilon^{\prime}<0\right)$ to metallic $\left(\varepsilon^{\prime}>0\right)$ and the second one, in the $\mathrm{CdO}$
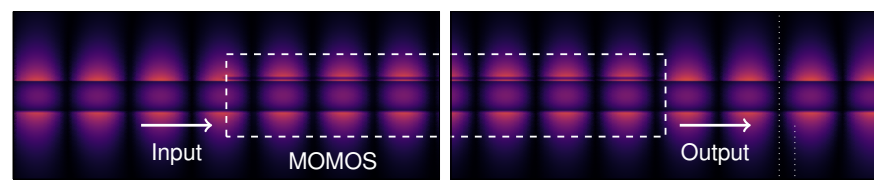

(a)
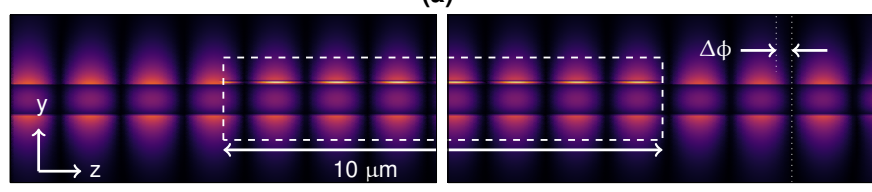

(b)

Fig. 6. Normalized TM electric field profile, $\left|E_{y}\right|$, obtained by $3 \mathrm{D}$ finite-difference time-domain (3D-FDTD) simulation at $\lambda=1550 \mathrm{~nm}$. Simulations were carried out for a $10-\mu \mathrm{m}-$ long MOMOS structure and a $\mathrm{CdO}$ floating gate carrier concentration of: (a) $N=2 \times 10^{19} \mathrm{~cm}^{-3}$ and (b) $N=2.3 \times 10^{20} \mathrm{~cm}^{-3}$.

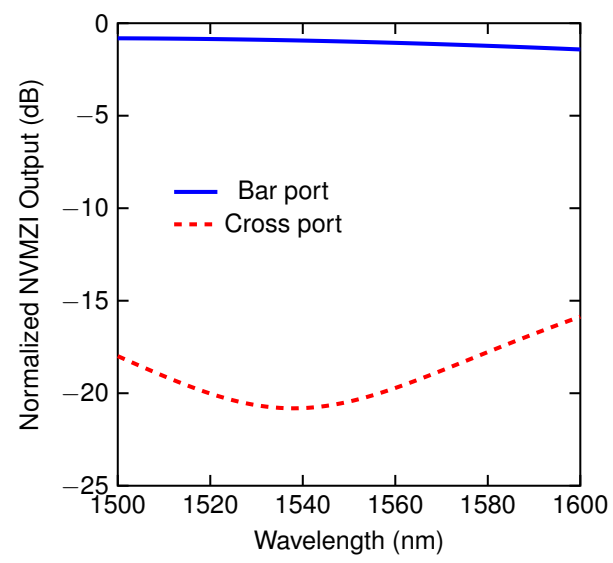

Fig. 7. Spectrum of the NVMZI when the floating gate of MOMOS bar arm is charged to $N=2.3 \times 10^{20} \mathrm{~cm}^{-3}$. The MOMOS length is $L_{\pi}$ and power input is at the bar port.

metallic region. The value of $L_{\pi}$ diminishes for higher values of $N$ with low-losses. However, to accumulate such as high carrier concentrations is not feasible. The minimum value of $L_{\pi}$ is found for $N \approx 3 \times 10^{20} \mathrm{~cm}^{-3}$, obtaining an $L_{\pi}$ below 10 $\mu \mathrm{m}$. However, optical losses are extremely large ( $>100 \mathrm{~dB})$ to be used as a phase shifter.

The threshold losses of the MOMOS is determined by setting the values of the extinction ratio and insertion loss of the NVMZI. These are shown in Fig. 5 as a function of the MOMOS losses at $\lambda=1550 \mathrm{~nm}$. In order to obtain more than $20 \mathrm{~dB}$ of extinction ratio with insertion loss lower than $1 \mathrm{~dB}$, the MOMOS must exhibit losses lower than $2 \mathrm{~dB}$. This criterion suffices from around $4 \times 10^{19} \mathrm{~cm}^{-3}$ to $2.3 \times 10^{20} \mathrm{~cm}^{-3}$ (see Fig. 4) in which the value of $L_{\pi}$ diminishes from $\sim 500 \mu \mathrm{m}$ to $31.75 \mu \mathrm{m}$, respectively. Therefore, since the device footprint is highly reduced for $N$ values near the ENZ regime, a target value of $2.3 \times 10^{20} \mathrm{~cm}^{-3}$ is chosen to charge the floating gate.

These results were confirmed by 3D finite-difference timedomain (3D-FDTD) simulations. A Si-MOMOS-Si waveguide with a short MOMOS structure was used in order to vary optical losses and the phase difference between both states. Fig. 6 shows the $\mathrm{x}$-cut of the TM optical mode for a 10- $\mu \mathrm{m}$-long MOMOS and when the $\mathrm{CdO}$ is discharged and charged to the aforementioned values. For the first case, optical losses are negligible (Fig. 6a). 

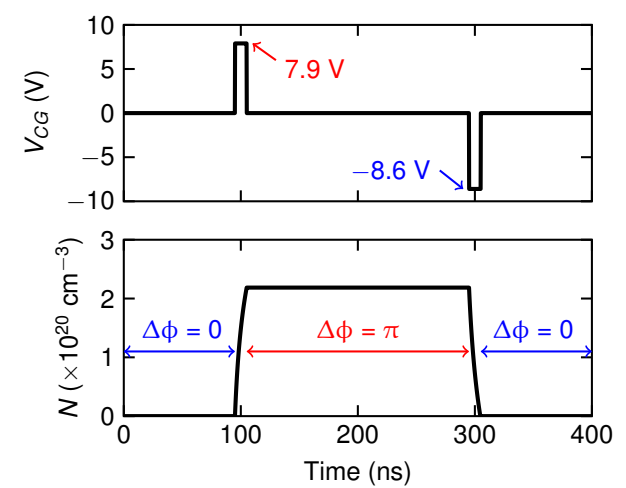

Fig. 8. Time domain evolution of $N$ as when both 10 ns wide charge and discharge electrical pulses are applied to the control gate.

On the other hand, when the floating gate is charged (Fig. 6b, the optical mode is mostly confined within the $\mathrm{CdO}$ in the MOMOS structure and thus, a phase shift is induced at the output. The phase increment is $\sim \pi / 3$, which agrees theory since the MOMOS length is three times shorter than $L_{\pi}$. Furthermore, optical losses are equal to $0.63 \mathrm{~dB}$ in agreement with those obtained by FEM simulations and thus, showing negligible coupling losses at the Si-MOMOS interface.

Finally, Fig. 7 shows the spectrum of the NVMZI when the floating gate of one of the arms is charged to $N=2.3 \times 10^{20}$ $\mathrm{cm}^{-3}$ and the MOMOS length is $L_{\pi}(\Delta \phi=\pi)$. The device shows an extinction ratio above $15 \mathrm{~dB}$ and insertion loss lower than $1.5 \mathrm{~dB}$ in a $100 \mathrm{~nm}$ range. When the floating gate is discharged insertion loss keeps negligible.

Regarding the non-volatile performance, Fig. 8 shows the time domain evolution of $N$ when both charge and discharge pulses are applied. On one hand, for a 10-ns-wide pulse, a control voltage of $7.9 \mathrm{~V}$ is required to charge the floating gate. Notice that, when the pulse is removed, the induced charge remains and thus, behaves with a non-volatile effect. On the other hand, a negative pulse is needed to discharge the floating gate. In this case, the required voltage value is a bit higher than in the charging process. This is due to the $\mathrm{Si} / \mathrm{Al}_{2} \mathrm{O}_{3}$ interface, which presents a lower energy band difference than the $\mathrm{CdO} / \mathrm{Al}_{2} \mathrm{O}_{3}$. These voltages give a power consumption per switch event of around $300 \mu \mathrm{W}$. The energy consumption is $\sim 3 \mathrm{pJ}$, which is comparable to silicon switches based on the plasma dispersion effect [22]. However, it should be noticed that the power consumption in the steady state is negligible in the proposed device. Finally, the time in which the device can maintain a $\Delta \phi=\pi$ is given by the retention time of the floating gate, which is predicted in the order of decades as in [11].

In conclusion, a non-volatile low-loss phase shifter has been proposed by using $\mathrm{CdO}$ as a floating gate in a flash-like structure. The phase shifter exhibits an active length to achieve a $\pi$ phase shift of around $30 \mu \mathrm{m}$ with an optical loss of $2 \mathrm{~dB}$. By integrating it in a symmetric Mach-Zehnder interferometer, a broadband non-volatile electro-optic switch can be attained with extinction ratios of $20 \mathrm{~dB}$ and insertion loss of $1 \mathrm{~dB}$. Furthermore, the device could be electrically controlled with voltage pulses below $10 \mathrm{~V}$ in the nanosecond range which leads to low- power and energy consumptions. These results could be used for implementing high-density non-volatile phase shifters in silicon photonic integrated circuits.
Funding. Ministerio de Economía y Competitividad (MINECO) (TEC2016-76849); Generalitat Valenciana (PROMETEO/2019/123); Ministerio de Ciencia, Innovación y Universidades (MICIU) (FPU17/04224).

Disclosures. The authors declare no conflict of interest.

\section{REFERENCES}

1. J. Feldmann, N. Youngblood, C. D. Wright, H. Bhaskaran, and W. H. Pernice, Nature 569, 208 (2019).

2. D. Pérez, I. Gasulla, L. Crudgington, D. J. Thomson, A. Z. Khokhar, K. Li, W. Cao, G. Z. Mashanovich, and J. Capmany, Nat. Commun. 8, 1 (2017).

3. C. Rios, M. Stegmaier, P. Hosseini, D. Wang, T. Scherer, C. D. Wright, H. Bhaskaran, and W. H. Pernice, Nat. Photonics 9, 725 (2015).

4. K. J. Miller, R. F. Haglund, and S. M. Weiss, Opt. Mater. Express 8, 2415 (2018).

5. C. A. Barrios and M. Lipson, J. Light. Technol. 24, 2898 (2006).

6. J. F. Song, X. S. Luo, A. E. J. Lim, C. Li, Q. Fang, T. Y. Liow, L. X. Jia, X. G. Tu, Y. Huang, H. F. Zhou, and G. Q. Lo, Sci. Reports 6, 1 (2016).

7. J.-F. Song, A. E. J. Lim, X. S. Luo, Q. Fang, C. Li, L. X. Jia, X. G. Tu, Y. Huang, H. F. Zhou, T. Y. Liow, and G. Q. Lo, Opt. Express 24, 21744 (2016).

8. M. Grajower, N. Mazurski, J. Shappir, and U. Levy, Laser Photonics Rev. 12, 1 (2018).

9. X. Li, N. Youngblood, C. Rios, Z. Cheng, W. H. P. Pernice, C. D. Wright, and H. Bhaskaran, Optica. 6, 1 (2019).

10. R. A. Soref and B. R. Bennett, IEEE J. Quantum Electron. 23, 123 (1987).

11. J. Parra, I. Olivares, A. Brimont, and P. Sanchis, Opt. Lett. 44, 3932 (2019).

12. V. E. Babicheva, N. Kinsey, G. V. Naik, M. Ferrera, A. V. Lavrinenko, V. M. Shalaev, and A. Boltasseva, Opt. Express 21, 27326 (2013).

13. Z. Ma, Z. Li, K. Liu, C. Ye, and V. J. Sorger, Nanophotonics 4, 198 (2015).

14. X. Liu, K. Zang, J. H. Kang, J. Park, J. S. Harris, P. G. Kik, and M. L. Brongersma, ACS Photonics 5, 4484 (2018).

15. N. Kinsey and J. Khurgin, Opt. Mater. Express 9, 2793 (2019).

16. S. Campione, M. G. Wood, D. K. Serkland, S. Parameswaran, J. Ihlefeld, T. S. Luk, J. R. Wendt, K. M. Geib, and G. A. Keeler, IEEE Photonics J. 9 (2017).

17. I. C. Reines, M. G. Wood, T. S. Luk, D. K. Serkland, and S. Campione, Opt. Express 26, 21594 (2018).

18. F. Hu, W. Jia, Y. Meng, M. Gong, and Y. Yang, Opt. Express 27, 26405 (2019).

19. F. Michelotti, L. Dominici, E. Descrovi, N. Danz, and F. Menchini, Opt. Lett. 34, 839 (2009).

20. S. K. Vasheghani Farahani, T. D. Veal, P. D. King, J. Zúñiga-Pérez, V. Muñoz-Sanjosé, and C. F. McConville, J. Appl. Phys. 109 (2011).

21. M. F. Limonov, M. V. Rybin, A. N. Poddubny, and Y. S. Kivshar, Nat. Photonics 11, 543 (2017).

22. A. Zanzi, C. Vagionas, A. Griol, A. Rosa, S. Lechago, M. MoralisPegios, K. Vyrsokinos, N. Pleros, J. Kraft, V. Sidorov, B. Sirbu, T. Telkin, P. Sanchis, and A. Brimont, Opt. Express 27, 32409 (2019). 